\title{
ORIGINAL ARTICLE Glucose deprivation increases monocarboxylate transporter 1 (MCT1) expression and MCT1-dependent tumor cell migration
}

\author{
CJ De Saedeleer, PE Porporato, T Copetti, J Pérez-Escuredo, VL Payen, L Brisson, O Feron and P Sonveaux
}

The glycolytic end-product lactate is a pleiotropic tumor growth-promoting factor. Its activities primarily depend on its uptake, a process facilitated by the lactate-proton symporter monocarboxylate transporter 1 (MCT1). Therefore, targeting the transporter or its chaperon protein CD147/basigin, itself involved in the aggressive malignant phenotype, is an attractive therapeutic option for cancer, but basic information is still lacking regarding the regulation of the expression, interaction and activities of both proteins. In this study, we found that glucose deprivation dose-dependently upregulates MCT1 and CD147 protein expression and their interaction in oxidative tumor cells. While this posttranslational induction could be recapitulated using glycolysis inhibition, hypoxia, oxidative phosphorylation (OXPHOS) inhibitor rotenone or hydrogen peroxide, it was blocked with alternative oxidative substrates and specific antioxidants, pointing out at a mitochondrial control. Indeed, we found that the stabilization of MCT1 and CD147 proteins upon glucose removal depends on mitochondrial impairment and the associated generation of reactive oxygen species. When glucose was a limited resource (a situation occurring naturally or during the treatment of many tumors), MCT1CD147 heterocomplexes accumulated, including in cell protrusions of the plasma membrane. It endowed oxidative tumor cells with increased migratory capacities towards glucose. Migration increased in cells overexpressing MCT1 and CD147, but it was inhibited in glucose-starved cells provided with an alternative oxidative fuel, treated with an antioxidant, lacking MCT1 expression, or submitted to pharmacological MCT1 inhibition. While our study identifies the mitochondrion as a glucose sensor promoting tumor cell migration, MCT1 is also revealed as a transducer of this response, providing a new rationale for the use of MCT1 inhibitors in cancer.

Oncogene (2014) 33, 4060-4068; doi:10.1038/onc.2013.454; published online 28 October 2013

Keywords: monocarboxylate transporter 1 (MCT1); basigin; glucose metabolism; mitochondrial dysfunction; reactive oxygen species (ROS); tumor cell migration

\section{INTRODUCTION}

Cancers evolve glycolysis as an aggressive metabolic phenotype allowing sustained energy production under hypoxia and unbridled cell proliferation when oxygen is present. ${ }^{1}$ From a metabolic standpoint, it is obvious that the optimal benefits of this glycolytic switch are reached in tumor areas where glucose is abundant and from which lactic acid, the end-product of glycolysis, is efficiently cleared. Otherwise, if glucose supply and/ or lactic acid removal fall below a minimum threshold, tumor cells (TCs) at distance from the vascular supply eventually die, which occurs naturally or during the course of therapy, for example, with vascular-disrupting or anti-angiogenic agents. Three strategies allow TCs to face inappropriate conditions for glycolysis: (i) to switch back to an oxidative metabolism (at the expense of proliferation and as long as enough oxygen and oxidative substrates are present), (ii) to migrate towards more favorable soils, or (iii) to manage to improve glucose delivery and lactate removal. The latter strategy largely depends on the paracrine use of lactate. ${ }^{2}$

Lactate shuttles first account for a metabolic symbiosis in tumors wherein oxygenated TCs import lactate to fuel oxidative phosphorylation (OXPHOS) through the tricarboxylic acid (TCA) cycle. ${ }^{3}$ The preferential use of lactate by these cells allows glucose to diffuse farther from blood vessels at a maximal theoretical rate of 1 glucose molecule spared for 2 molecules of lactate used. It primarily depends on the expression and activity of monocarboxylate transporter 1 (MCT1), a passive lactate-proton symporter facilitating lactic acid uptake by oxidative TCs (whereas MCT4 channels lactic acid out of glycolytic TCs). ${ }^{2,4,5}$ Since lactate shuttling is coupled with one proton, the use of MCT1 to fuel OXPHOS with lactate opposes extracellular acidification. A second contribution of lactate to the metabolic optimization of the tumor microenvironment is referred to as 'lactate signaling'. In oxidative TCs and in endothelial cells, it shares with the metabolic lactate pathway the dependency on MCT1 and the need to oxidize lactate into pyruvate, but requires only a low rate of lactate uptake. ${ }^{6-8}$ While TCs increase their production of vascular endothelial growth factor in response to lactate, 8,9 endothelial cells are triggered to upregulate the expression of vascular endothelial growth factor receptor 2, basic fibroblast growth factor, and interleukin-8, ${ }^{6-8}$ thus providing several paracrine and autocrine pathways through which lactate induces angiogenesis. Additional activities of lactate in tumors have been reviewed recently. ${ }^{2}$ Collectively, the use of lactate as a fuel and as a signaling agent offers an aggressive advantage to tumors, which is clinically supported by the positive correlation observed between high levels of lactate and tumor progression in several types of human tumors. ${ }^{10}$ Conversely, MCT1 inhibition has been well documented preclinically to exert

Pole of Pharmacology, Institut de Recherche Expérimentale et Clinique (IREC), Université catholique de Louvain (UCL), Brussels, Belgium. Correspondence: Professor P Sonveaux, Pole of Pharmacology, Institut de Recherche Expérimentale et Clinique (IREC), Université catholique de Louvain (UCL), Avenue Emmanuel Mounier 53 box B1.53.09, 1200, Brussels, Belgium.

E-mail: pierre.sonveaux@uclouvain.be

Received 19 December 2012; revised 4 July 2013; accepted 16 September 2013; published online 28 October 2013 
antimetabolic and anti-angiogenic effects leading to hypoxic/ glycolytic TC death by virtue of glucose starvation. ${ }^{3,6-8}$ A first orally-available MCT1 inhibitor having demonstrated preclinical safety is currently entering into clinical trials. ${ }^{1}$

Despite the fact that MCT1 has emerged as a new anticancer target, there is only limited general knowledge about the molecular mechanisms governing its expression and activity. In the exercising muscle, transcriptional induction is exerted through calcium and AMP, as recently reviewed by Halestrap and Wilson. ${ }^{11}$ In cancer cells, gene expression may further be regulated by promoter methylation/inactivation. ${ }^{12}$ At the posttranslational level, the membrane expression and transport activities of MCT1 require its direct interaction with the scaffold protein CD147. ${ }^{13-15}$ The assembly of the complex exerts a stabilizing effect, which is necessary for the trafficking, cell surface localization and function of both proteins. In this study, we focused on tumor parameters and report that glucose deprivation posttranslationally stabilizes both MCT1 and CD147. This response molecularly involves reactive oxygen species (ROS) produced as a result of mitochondrial dysfunction. The consequent formation of functional MCT1-CD147 complexes confers to TCs the ability to evade a glucose-depleted environment. This study collectively provides an unprecedented pathway linking mitochondrial ROS production to the stimulation of MCT1-dependent TC migration towards glucose.

\section{RESULTS}

Glucose deprivation stabilizes MCT1 and CD147 protein expression in tumor cells

Limited glucose bioavailability is a key feature of the tumor microenvironment ${ }^{16,17}$ that we tested as a potential modulator of MCT1 and CD147 expression. It was experimentally induced by depriving cells of glucose in a medium containing no glutamine, pyruvate, lactate or serum, and an equal amount of viable TCs were systematically compared. As models, we used SiHa cells that were previously documented to rely on OXPHOS for energy production $^{3,8}$ and HeLa cells known for their ability to switch from a glucose to a glutamine- (i.e., OXPHOS-dependent) fueled metabolism. $^{18}$ In a first series of experiments, we observed a dose-dependent increase in MCT1 and CD147 protein expression in SiHa cells cultured during $48 \mathrm{~h}$ with decreasing glucose concentrations (Figure 1a). This inverse relationship was confirmed statistically by showing that glucose starvation caused a a
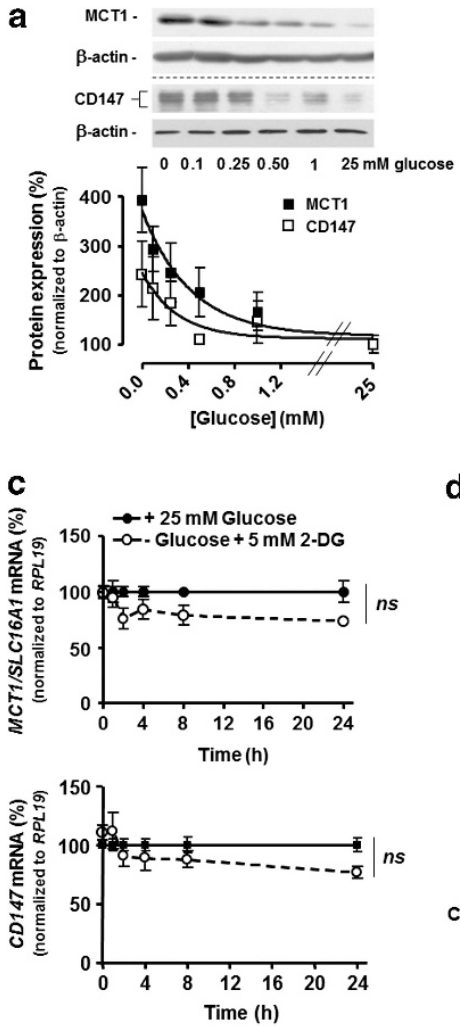

b
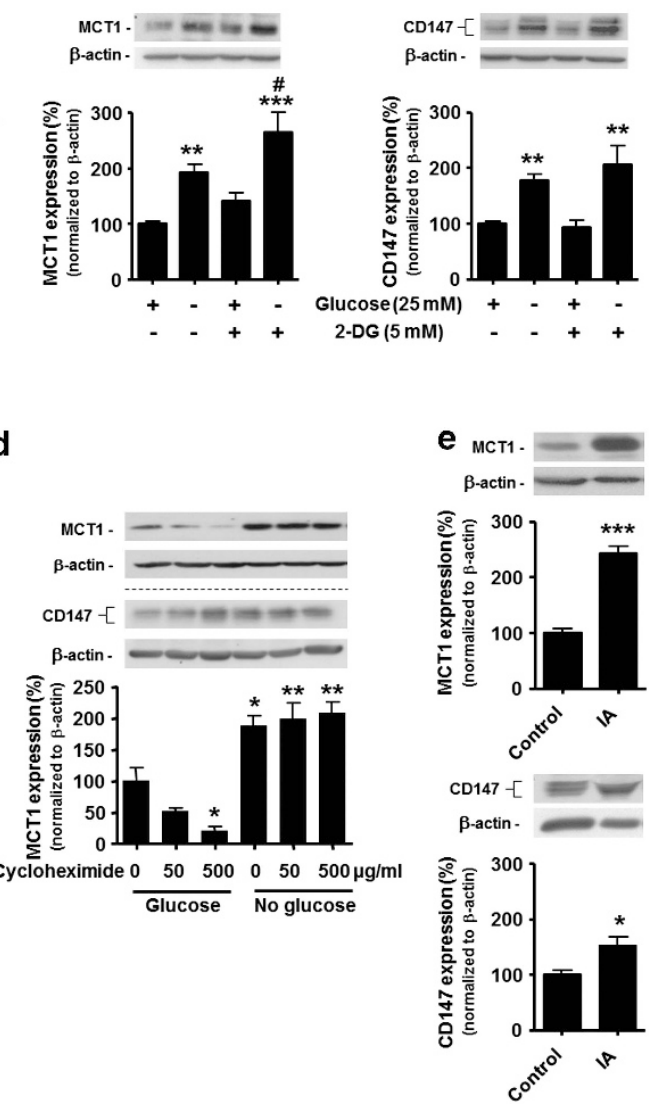

Figure 1. Impaired glycolysis induces MCT1 and CD147 expression. (a) Representative immunoblots and the bar graph represent MCT1 and CD147 protein expression in oxidative SiHa cells cultured during $48 \mathrm{~h}$ with the indicated concentrations of glucose, without glutamine, pyruvate, lactate or serum. $\beta$-actin was used as a loading control $(n=3)$. (b) SiHa cells were cultured during $24 \mathrm{~h}$ in the presence of glucose and of the hexokinase competitor 2-deoxyglucose (2-DG) where indicated. Representative immunoblots and bar graphs represent MCT1 (left) and CD147 (right) protein expression normalized by $\beta$-actin ${ }^{* *} P<0.01$, ${ }^{* * *} P<0.005$ compared to medium with glucose without 2-DG; ${ }^{\#} P<0.05$ compared to medium without glucose and 2-DG; $n=3-6$ ). (c) SLC16A1/MCT1 (top) and CD147 (bottom) mRNA in SiHa cells cultured during the indicated times in the presence of glucose and 2-DG where indicated (ns, not significant; $n=3$ ). (d) SiHa cells were cultured during $24 \mathrm{~h}$ in the presence of glucose and of protein translation inhibitor cycloheximide where indicated. Representative immunoblots are shown for MCT1 and CD147, and the bar graph represents MCT1 protein expression normalized by $\beta$-actin. $\left({ }^{*} P<0.05\right.$, ${ }^{* *} P<0.01$ compared to medium with glucose without cycloheximide; $n=3$ ). (e) Representative immunoblots and the bar graph show MCT1 (top) and CD147 (bottom) protein expression in SiHa cells cultured during $24 \mathrm{~h}$ with glucose and the GAPDH inhibitor 2-iodoacetate (IA) where indicated $\left({ }^{*} P<0.05,{ }^{* * *} P<0.005, n=3\right)$. 
significant increase in the expression of both proteins in $\mathrm{SiHa}$ (Figure 1b) and in HeLa (Supplementary Figure 1A) cells. In these experimental settings with $\mathrm{SiHa}$, the glucose transporter and hexokinase competitor 2-deoxyglucose (2-DG, $5 \mathrm{~mm}, 24 \mathrm{~h}$ ) did not recapitulate the response to glucose starvation but it significantly increased MCT1 expression in the absence of glucose (Figure 1b). We then focused on the mechanism. When compared to measurements in the presence of glucose, glucose withdrawal with 2-DG did not significantly modulate SLC16A1/MCT1 and CD147 mRNA expression over time (Figure 1c), ruling out a transcriptional effect. It rather stabilized both proteins posttranslationally. Indeed, the addition of cycloheximide ( $24 \mathrm{~h})$ caused a decrease in MCT1 protein expression in the presence of glucose but not under glucose starvation where higher MCT1 levels were systematically detected (Figure 1d). Induction of CD147 was similarly preserved (Figure 1d) although cycloheximide also prolonged the expression of the protein in the presence of glucose, indicating that CD147 has a longer half-life than MCT1 in SiHa cells. Increased MCT1 and CD147 protein expression was observed when culturing the cells with the irreversible glyceraldehyde-3-phosphate dehydrogenase (GAPDH) inhibitor 2-iodoacetate $\left(200 \mu \mathrm{m}, 24 \mathrm{~h}\right.$, Figure 1e). ${ }^{19}$ A similar response was observed in HeLa cells (Supplementary Figure 1B). These data collectively indicate that disruption of glycolysis increases MCT1 and CD147 protein stability in oxidative TCs.

In the absence of exogenous glutamine, pyruvate, lactate and serum, glucose removal logically caused time-dependent $\mathrm{SiHa}$ cell death (Supplementary Figure 2A). This and the well-known property of glucose deprivation to promote mutations in $\mathrm{TCs}^{20}$ prompted us to test whether clonal selection could account for changes in MCT1 and/or CD147 expression. It was ruled out by showing i) that the reintroduction of glucose after a starvation period of $24 \mathrm{~h}$ (resulting in increased MCT1 and CD147 protein expression) restored basal protein expression levels (Supplementary Figure 2B), and ii) that the glucose starvation of 2 independent clones isolated from the total $\mathrm{SiHa}$ population caused a significant increase in the expression of both proteins (Supplementary Figures 2C-D).

Mitochondrial activity controls MCT1 and CD147 expression in tumor cells

Lack of glucose or inhibition of glycolysis reduces mitochondrial anaplerosis. A potential involvement of mitochondria in the control of MCT1 and CD147 expression was first addressed by showing that hypoxia $\left(1 \% \mathrm{O}_{2}, 24 \mathrm{~h}\right)$ triggers the expression of both proteins in $\mathrm{SiHa}$ (Figure 2a) and in HeLa (Supplementary Figure 1B) cells. Glucose deprivation, glycolysis inhibition and hypoxia share the common property of impairing OXPHOS. We therefore tested whether direct OXPHOS inhibition could impact MCT1 and CD147 protein expression by treating $\mathrm{SiHa}$ cells with the respiratory chain complex I inhibitor rotenone $(1 \mu \mathrm{g} / \mathrm{ml})$. Rotenone induced a $\sim 2$-fold and a 1.4-fold increase in MCT1 and CD147 expression, respectively (Figure 2b). Similar data were obtained with HeLa cells (Supplementary Figure 1B). To test whether restoring OXPHOS could repress the increase in MCT1 and CD147 protein expression, we took advantage of our glucose starvation model. $\mathrm{SiHa}$ cell death upon glucose removal was rescued with lactate (Supplementary Figure 2A), a substrate known to efficiently replace glucose as an oxidative fuel for these cells. ${ }^{3}$ Western blots further showed that lactate totally suppressed the induction of MCT1 and CD147 expression that was otherwise detected $48 \mathrm{~h}$ after glucose removal (Figure 3a). It was confirmed using the two $\mathrm{SiHa}$ clones (Supplementary Figure 2E) and HeLa cells (Supplementary Figure 2F). Conversely, lactate in the presence of glucose did not change the expression of both proteins (Supplementary Figures $3 \mathrm{~A}, \mathrm{~B}$ showing that increasing doses of lactate did not modulate MCT1 expression in the presence of a
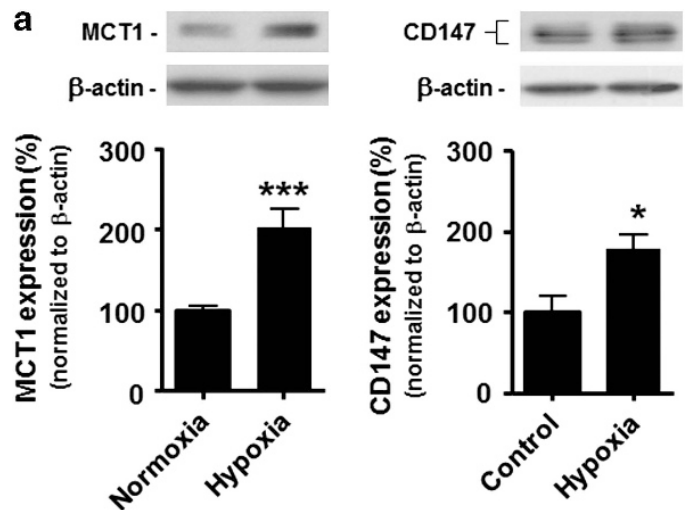

b
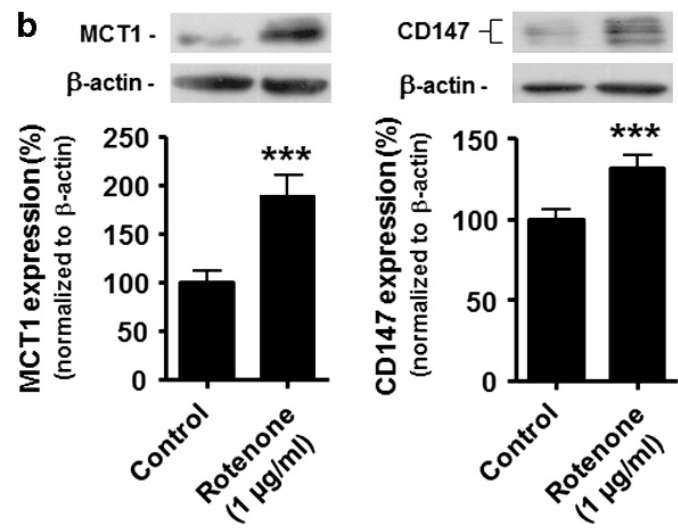

Figure 2. Hypoxia and OXPHOS inhibition induce MCT1 and CD147 expression. (a) Representative immunoblots and the bar graph show MCT1 (left) and CD147 (right) protein expression in SiHa cells cultured during $24 \mathrm{~h}$ under hypoxia $\left(1 \% \mathrm{O}_{2}\right)$ or not. $\beta$-actin was used as a loading control. $\left({ }^{*} P<0.05,{ }^{* * *} P<0.005, n=5-6\right)$. (b) SiHa cells in glucose-containing medium were treated with rotenone or not during $48 \mathrm{~h}$. Pictures show representative immunoblots and the graphs MCT1 (left) and CD147 (right) protein expression normalized by $\beta$-actin $(* * * P<0.005, n=9-10)$.

glucose). As with lactate, the delivery of the oxidative substrate glutamine to glucose-starved SiHa cells blunted MCT1 and CD147 protein upregulation, whereas glutamine did not exert such influence in the presence of glucose (Figure 3b). In fact, refueling mitochondria prevented the response to glucose starvation irrespectively of the molecular nature of the fuel. This was further demonstrated using the cell-permeable esterified forms of the TCA cycle substrates pyruvate and succinate: in the absence of glucose, either methyl-pyruvate or dimethyl-succinate successfully blocked the induction of MCT1 and CD147 protein expression in $\mathrm{SiHa}$ (Figure 3c) and in HeLa (Supplementary Figure 2G) cells. In the presence of glucose, these compounds did not modulate the basal expression of both proteins (Supplementary Figure 3C).

Mitochondrial ROS mediate the induction of MCT1 and CD147 protein expression upon glucose starvation

Dysfunctional mitochondria are a main source of ROS, among which superoxide is the initial species. ${ }^{21}$ It can be measured in intact cells using flow cytometry with MitoSOX, a superoxidespecific fluorogenic probe with a high tropism for mitochondria. ${ }^{22}$ Glucose starvation (Figures $4 a$ and b), glycolysis inhibition with iodoacetate (Figure 4c) and rotenone (Figure 4d) at the doses used in our previous experiments all independently triggered superoxide production in SiHa cells. More particularly, we documented a $\sim 3$-fold increase in the mitochondrial superoxide production of 
a
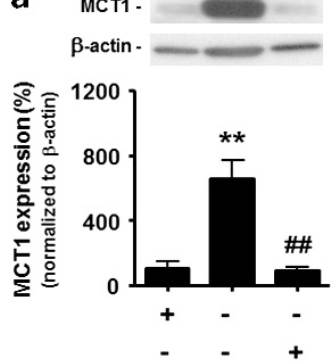

b
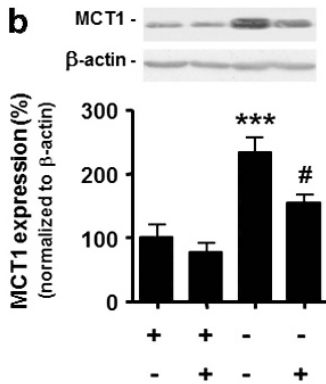

c
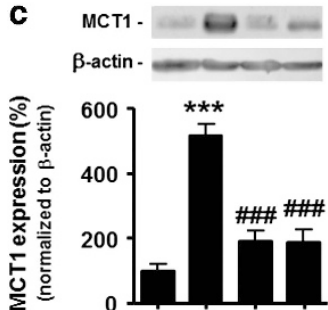
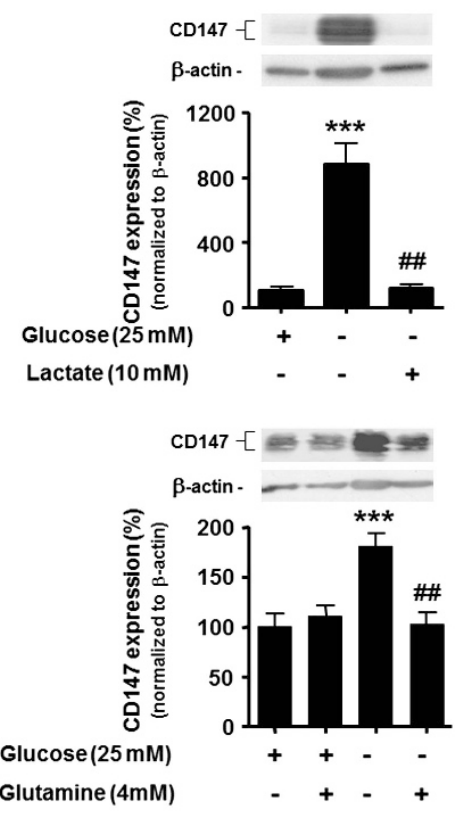

Glutamine (4mM)

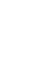

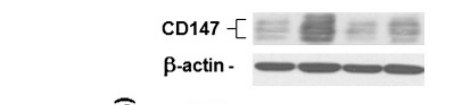
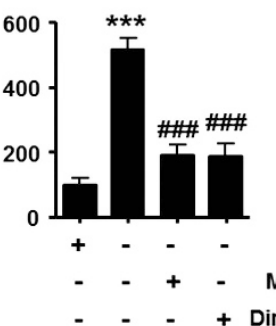

Glucose $(25 \mathrm{mM})$

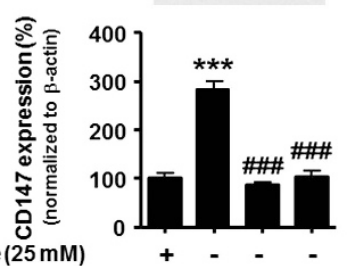

Methyl-pyruvate (10 mM) - - + -

Figure 3. In the absence of glucose, alternative oxidative fuels prevent MCT1 and CD147 overexpression. (a-c) SiHa cells were cultured during $48 \mathrm{~h}$ under glucose starvation or not. Pictures show representative immunoblots. Left graphs depict MCT1 expression. Right graphs show CD147 expression. $\beta$-actin was used as a loading control. (a) Lactate was supplied where indicated ${ }^{* *} P<0.01$, ${ }^{* * *} P<0.005$ compared to medium containing glucose but no lactate; ${ }^{\# \#} P<0.01$ compared to glucose- and lactate-deprived medium; $n=3$ ). (b) Glutamine was supplied where indicated $\left({ }^{* * *} P<0.005\right.$ compared to medium containing glucose but no glutamine; ${ }^{\#} P<0.05,{ }^{\# \#} P<0.01$ compared to glucose- and glutaminedeprived medium; $n=6$ ). (c) Methyl-pyruvate or dimethyl-succinate was supplied where indicated $(* * * P<0.005$ compared to medium supplied only with glucose; ${ }^{\# \#} P<0.005$ compared to glucosedeprived basal medium; $n=3$ ).

glucose-starved compared to glucose-fueled SiHa cells (Figures 4a and b). Lactate in the absence of glucose prevented the increase in superoxide production (Figure 4a) as also did the more direct TCA cycle intermediates methyl-pyruvate and dimethyl-succinate (Figure 4b), correlating with the MCT1 and CD147 protein data shown in Figures $3 a$ and $c$, respectively.

That ROS can stimulate MCT1 and CD147 protein expression was established by showing a $\sim 2$-fold increase in the expression of both proteins $24 \mathrm{~h}$ after treatment with $0.8 \mathrm{~mm} \mathrm{H}_{2} \mathrm{O}_{2}$ (Figure 4e). In line with what was observed upon glucose removal (Figure 1c), we did not detect significant changes in the abundance of the corresponding transcripts (Figure 4f). Furthermore, as with glucose starvation (Figure 1d), the induction of both proteins was preserved upon treatment with cycloheximide (Figure 4g), revealing that glucose withdrawal and ROS share the ability to stabilize
MCT1 and CD147 proteins posttranslationally. We therefore sought to document a direct link. The general antioxidant and glutathione analogue $\mathrm{N}$-acetyl-L-cysteine (NAC, $5 \mathrm{~mm}$ ) blocked MCT1 and CD147 overexpression in glucose-starved SiHa cells (Figure 4h). A similar effect was observed with the mitochondriaselective superoxide scavenger MitoTEMPO $(50 \mu \mathrm{m})^{23}$ (Figure 4i), thus confirming that ROS produced by mitochondria when they are deprived from glycolytic fuels trigger the expression of both the lactate transporter and its chaperone. ROS were also key contributors to the response to hypoxia: NAC or the $\mathrm{H}_{2} \mathrm{O}_{2}$ scavenger ebselen $(20 \mu \mathrm{m})^{24}$ both blocked hypoxia-induced MCT1 expression (Supplementary Figure 4A). None of the antioxidants influenced MCT1 and CD147 protein expression in glucose-fueled, normoxic TCs (Supplementary Figures 4A-C).

Glucose starvation promotes the formation of MCT1-CD147 heterocomplexes at the plasma membrane of tumor cells

As MCT1 and CD147 underwent a concerted regulation in all experimental conditions, it was important to address the formation of functional heterocomplexes. Information was provided using immunocytochemistry: glucose deprivation dosedependently caused an increase in the expression of MCT1 and CD147 at the plasma membrane of SiHa cells where both proteins colocalized (Supplementary Figure 5A). Direct protein-protein interaction was imaged using proximity ligation assay. SiHa cells incubated in a glucose-deprived medium during $24 \mathrm{~h}$ showed a $\sim 2$-fold increase in MCT1-CD147 interactomes compared with cells incubated in glucose-containing medium (Figure $5 \mathrm{a}$ ). In both treatment conditions, heterocomplexes were located in the cytosol and at the plasma membrane, including in F-actin-positive protrusions, and their abundance increased at all locations in the absence of glucose (Figure 5b and Supplementary Figure 5B).

MCT1 facilitates tumor cell migration towards serum and glucose Filipodia are sensory organelles used by invasive cancer cells to probe their microenvironment during migration. ${ }^{25}$ These cell protrusions are characterized by the presence of actin filaments. Upon glucose starvation, the apparent increase in MCT1-CD147 heterocomplexes in such structures among other locations (Figure $5 \mathrm{~b}$ and Supplementary Figure $5 \mathrm{~B}$ ) prompted us to test the contribution of MCT1 to TC migration. In transwell assays, we first documented a pro-migratory role of MCT1 towards serum: in our experimental settings, targeting MCT1 with two different specific shRNAs impaired SiHa and HeLa cell migration by $\sim 2$ - to 4-fold compared to the cells infected with a control shRNA (Figure 6a, target extinction is shown in Supplementary Figure 6A). Based on this, we functionally assayed the metabolic control of MCT1 and CD147 expression and interaction by studying TC migration towards glucose. In transwells, shCTR-expressing $\mathrm{SiHa}$ (Figure $6 \mathrm{~b}$ ) or HeLa (Figure $6 \mathrm{c}$ ) cells starved from glucose in the upper well migrated towards glucose present in the lower well. In the absence of MCT1, the specific migration of both cell lines towards glucose was totally inhibited (Figures $6 \mathrm{~b}$ and c). Next, we showed that the addition of either glucose or lactate to the upper well also significantly inhibited SiHa migration (Figure $6 \mathrm{~d}$ ), thus providing a functional coupling between the lack of oxidative substrates and the migration of oxidative TCs towards glucose. There was no increase in random cell migration when glucose was absent in both wells of the assay compared to the situation where glucose was present in both, and lactate did not impact the migration of glucose-fueled $\mathrm{SiHa}$ cells (Figure $6 \mathrm{~d}$ ). It is of note that, contrary to glucose, neither lactate nor glutamine acted as a chemoattractant for $\mathrm{SiHa}$ cells in our experimental conditions (Supplementary Figure 6B).

The involvement of ROS in the migratory phenotype was probed with NAC (5 $\mathrm{mm})$, which in transwells inhibited the specific but not the basal migration of $\mathrm{SiHa}$ and HeLa cells towards 
a
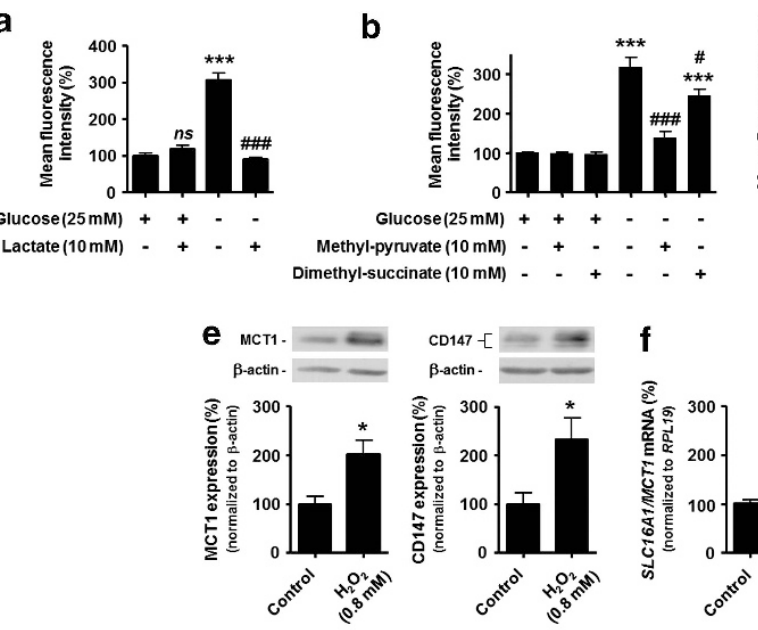

$\mathbf{f}$
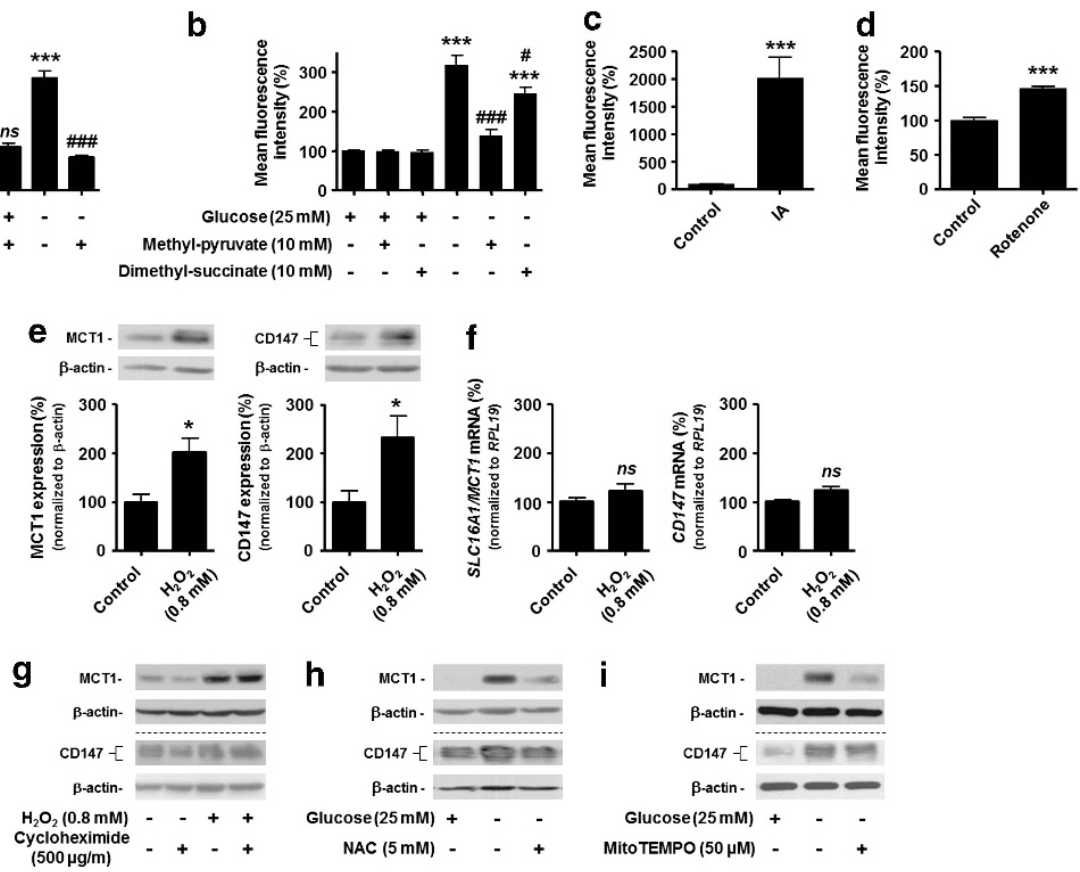

Figure 4. Mitochondrial ROS mediate the upregulation of MCT1 and CD147 in glucose-starved tumor cells. (a-d) Flow cytometry and the fluorogenic probe MitoSOX ${ }^{22}$ were used to detect mitochondrial superoxide in SiHa cells. Histograms show the mean fluorescence intensity of independent measurements with 50000 cells (\%). Cells were cultured during $24 \mathrm{~h}$ with, where indicated, (a) glucose and sodium L-lactate (ns, $P>0.05,{ }^{* * *} P<0.005$ compared to medium containing glucose but no lactate; ${ }^{\# \# \# ~} P<0.005$ compared to glucose- and lactate-deprived medium), (b) glucose, methyl-pyruvate and/or dimethyl-succinate ( ${ }^{* *} P<0.005$ compared to medium supplied only with glucose; ${ }^{\#} P<0.05$, ${ }^{\# \# \#} P<0.005$ compared to glucose-deprived basal medium; $\left.n=4\right)$, (c) 2 -iodoacetate $(\mathrm{IA}, 200 \mu \mathrm{m})(* * * P<0.005, n=4-5)$, or $(\mathbf{d})$ rotenone $(1 \mu \mathrm{g} / \mathrm{ml})$ $\left({ }^{* *} P<0.005, n=4-5\right)$. Glucose $(25 \mathrm{~mm})$ was systematically present in (c) and (d). (e) Immunoblots and the bar graph represent MCT1 (left) and CD147 (right) protein expression in glucose-supplied SiHa cells cultured during $24 \mathrm{~h}$ with or without $\mathrm{H}_{2} \mathrm{O}_{2}$. $\beta$-actin was used as a loading control. ( $\left.{ }^{*} P<0.05, n=6\right)$. (f) Same treatments as in (e) but detecting SLC16A1/MCT1 (left) and CD147 (right) mRNA expression (ns, not significant, $n=3$ ). (g-i) Immunoblots are representative of 3 independent experiments and show MCT1, CD147 and $\beta$-actin expression in SiHa cells after $48 \mathrm{~h}$ of culture with, where indicated, $(\mathbf{g}) \mathrm{H}_{2} \mathrm{O}_{2}$ and cycloheximide always in the presence of glucose ( $25 \mathrm{~mm}$ ), (h) glucose and $\mathrm{N}$-acetyl-L-cysteine (NAC), or (i) glucose and the mitochondrial-selective superoxide scavenger MitoTEMPO. ${ }^{23}$ In (h and i), low exposition of the film was used to show the antioxidant effect on MCT1; MCT1 expression with glucose alone was detected with longer exposure times (not shown).

glucose (Figure 6e). Thus, ROS are key mediators of the migration of glucose-deprived TCs towards glucose. MCT1 or CD147 overexpression was sufficient to increase $\mathrm{SiHa}$ cell migration, and the increased migratory phenotype was insensitive to NAC inhibition (Figure $6 \mathrm{f}$, with serum as chemoattractant). Because MCT1 overexpression stabilized CD147 protein expression and vice versa (Supplementary Figure $6 \mathrm{C}$ ) and given that migration was equally inhibited by removing MCT1 or ROS, our experiments collectively indicated that MCT1-CD147 heterocomplexes are key downstream effectors of mitochondrial ROS in the promigratory phenotype of glucose-starved TCs. MCT1 was an active contributor, as illustrated by the fact that $\alpha$-cyano-4-hydroxycinnamate, a drug known to reversibly inhibit the transport activity of MCT1 with a 10 -fold selectivity over other MCTs, ${ }^{26}$ completely blocked the migration of glucose-starved $\mathrm{SiHa}$ and HeLa cell towards glucose (Figure $6 \mathrm{~g}$ ). It did not influence the random migration of glucosefueled TCs.

\section{DISCUSSION}

While the monocarboxylate transporter MCT1 has emerged as a new anticancer target and a first MCT1 inhibitor is currently entering clinical trials, ${ }^{1,2}$ little is known about its regulation by typical parameters of the tumor microenvironment. ${ }^{11,12,14}$ This basic information, however, is essential for the prediction of treatment sensitivity and resistance as well as for the rational design of combination therapies. In this study, we report that mitochondria deprived from glycolytic fuels stabilize the expression of MCT1 and of its chaperone protein CD147/basigin in human TCs, thus providing a functional complex known to be involved in the transport of monocarboxylic acids (through MCT1) and in the activation of matrix metalloproteinases (through CD147). ${ }^{11,14,15}$ Upon glucose starvation, the complex promoted TC migration towards serum and glucose. Our study identifies a functional link between impaired mitochondrial activity and TC migration wherein the mitochondrion acts as a glucose sensor and signals via ROS MCT1-dependent TC escape to a more favorable metabolic environment.

A disruption of the functional coupling between the TCA cycle and OXPHOS is a fundamental metabolic characteristic of cancer. ${ }^{27}$ It is primarily achieved when TCs outgrow their oxygen and nutrient supply, marking the switch to a glycolytic metabolism. It is also typical of TCs constitutively addicted to glycolysis (the Warburg Effect). ${ }^{1}$ Here, using well-characterized oxidative human $\mathrm{TCs}^{3,8}$ we observed that both glucose starvation and hypoxia stimulate MCT1 and CD147 expression in vitro (Figures 1 and 2). These conditions are typical of the tumor microenvironment. While hypoxia has been extensively characterized to be naturally associated with tumor progression, fewer yet convincing data are currently available reporting natural glucose depletion in tumors. In experimental settings, Walenta et al. ${ }^{16}$ used bioluminescence to evidence a progressive decline of glucose towards the core of rat mammary tumors. Others using capillary electrophoresis coupled to mass spectrometry reported levels of glucose as low as $123 \pm 43 \mathrm{nmol} / \mathrm{g}$ and $424 \pm 131 \mathrm{nmol} / \mathrm{g}$ in patient biopsies of colon and stomach cancer, respectively. ${ }^{17}$ 
a

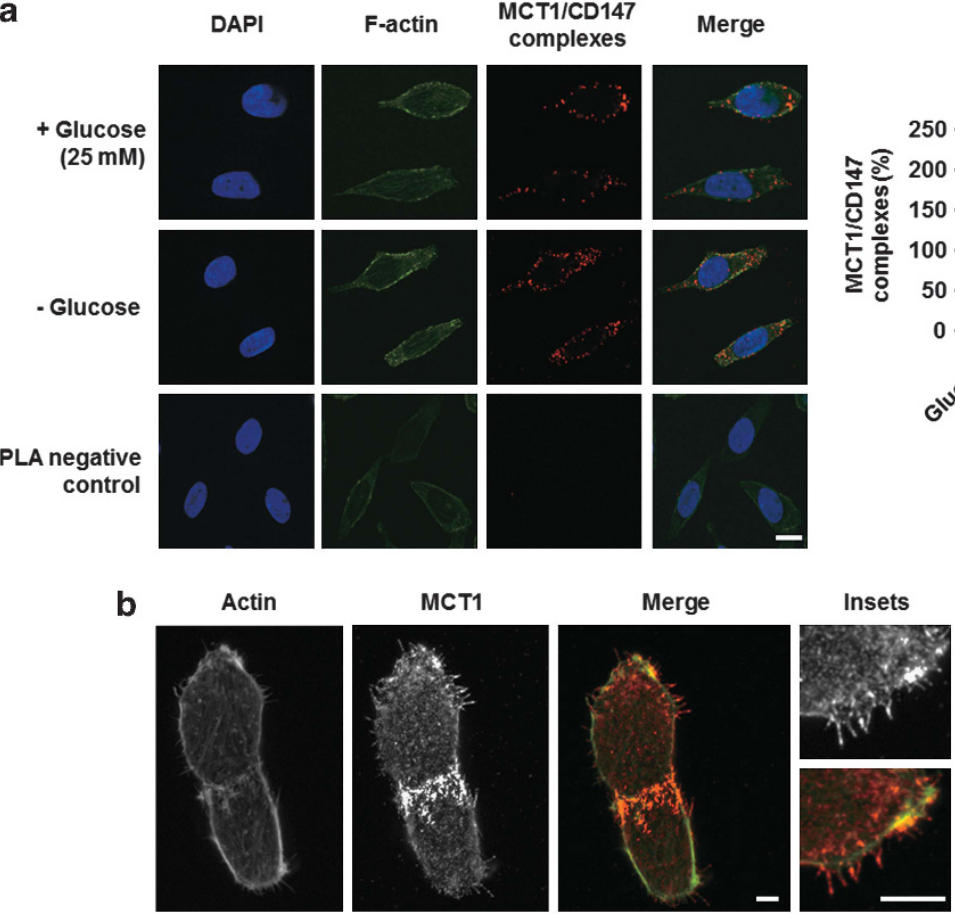

Figure 5. Glucose starvation promotes the formation of MCT1-CD147 heterocomplexes. (a) SiHa cells were cultured during $24 \mathrm{~h}$ in the presence of glucose or not. MCT1-CD147 heterocomplexes were detected using a proximity ligation assay (PLA) and appear as red dots in the representative pictures where cell nuclei are in blue (DAPI) and F-actin is in green (phalloidin). Omission of the primary antibody against CD147 was used as negative control. Bar $=10 \mu \mathrm{m}$. The number of interactomes was quantified in $42-50$ cells per treatment condition and is expressed as \% in the graph $(* * * P<0.005)$. (b) Fluorescent immunolabeling of MCT1 and the staining of F-actin (phalloidin) are shown in a dividing SiHa cell. Inset shows F-actin-positive membrane protrusions that stained positively for MCT1. Bars $=5 \mu \mathrm{m}$.

These concentrations were 45-times (colon) and 13-times (stomach) lower than that in the corresponding normal tissues, supporting the existence of a steep natural gradient of glucose deprivation in clinical tumors. Glucose and oxygen deprivation is further relevant to cancer therapy ${ }^{28}$ and the primary aim of antiangiogenic and antivascular treatments. According to our in vitro results (Figure 1 and Supplementary Figure 5), limited glucose availability can stabilize MCT1-CD147 complexes in a dosedependent manner and, consequently, promotes MCT1-dependent TC migration (Figure 6). The effect is posttranslational (Figure 1d) and as such may involve a reduced turnover rate of both proteins known to be subject to endosome-dependent recycling. ${ }^{29,30}$ These responses to low glucose further explain the metastatic stimulation that can be associated with the use of anti-angiogenic drugs ${ }^{31}$ and the higher expression of MCT1 in metastatic lesions compared to primary tumors in patients. ${ }^{32}$ Conversely, increased MCT1 expression promotes the anticancer activity of 3bromopyruvate, ${ }^{33}$ an alkylating agent strictly dependent on MCT1 for its uptake, ${ }^{34}$ thus offering a new rationale for combination therapy with anti-angiogenic or antivascular drugs.

In the absence of other nutrients, limited glucose availability deprives mitochondria of essential fuels and promotes oxidative stress. ${ }^{35}$ Cell survival then relies on autophagy and the ability to counter excess ROS production. Here, we show that these survivor cells overexpress MCT1 and CD147 independently of clonal selection. It may therefore be viewed as an integral part of cell adaptation to low glucose. The molecular response was mimicked by inhibiting glycolysis in otherwise oxidative TCs (Figure 1e and Supplementary Figure 1B) or by inducing OXPHOS inhibition (Figure 2). Others have recently shown that glutamine deprivation enhances MCT1 expression in TCs. ${ }^{33}$ Conversely, MCT1 and CD147 overexpression in glucose-starved TCs was repressed with replacement oxidative fuels (Figure 3 and Supplementary
Figures $2 \mathrm{E}-\mathrm{G})$. These data collectively indicate that mitochondria function as glucose sensors and signaling organelles, maintaining low MCT1-CD147 expression when functioning in the OXPHOS mode but stimulating the expression of both proteins upon direct or indirect OXPHOS impairment.

While the migration of glucose-starved TCs depends on MCT1 expression and activity (Figure 6), CD147 is known to promote invasion, ${ }^{15,36,37}$ so it can logically be proposed that MCT1-CD147 accumulation allows cell to escape from harsh environments. Accordingly, forced overexpression of either of the partners (resulting in the stabilization of both of them as previously reported in other systems) ${ }^{13-15}$ was sufficient to promote TC migration (Figure $6 \mathrm{f}$ and Supplementary Figure $6 \mathrm{C}$ ). The contribution of MCT1 to migration was seen with glucose or serum as chemoattractants but not with lactate or glutamine used at their relevant concentrations in culture media (Supplementary Figure 6B), explaining why others in non-metabolically controlled systems failed to detect MCT1-dependent migration other than random. ${ }^{37}$ On another hand, CD147 upregulation under glucose starvation could help survivor cells to combat excess oxidative stress before evading to better soils: in malignant melanoma cells, silencing CD147 has been shown to induce ROS production. ${ }^{38}$

Our characterization of the molecular pathway through which mitochondria control $\mathrm{MCT1}$ and $\mathrm{CD} 147$ revealed a key role of mitochondrial ROS. Glucose depletion is well known to trigger ROS production as a result of electron leakage at the electron transport chain, ${ }^{35}$ and did so in our experimental settings (Figure 4). While exogenous $\mathrm{H}_{2} \mathrm{O}_{2}$ stabilized both MCT1 and CD147 protein expression posttranslationally thus mimicking glucose deprivation, TCA cycle replenishment upon glucose withdrawal reduced mitochondrial ROS production and inhibited protein stabilization, phenocopying antioxidants (Figures 3 and 4). The repressor effect of the specific mitochondrial superoxide 


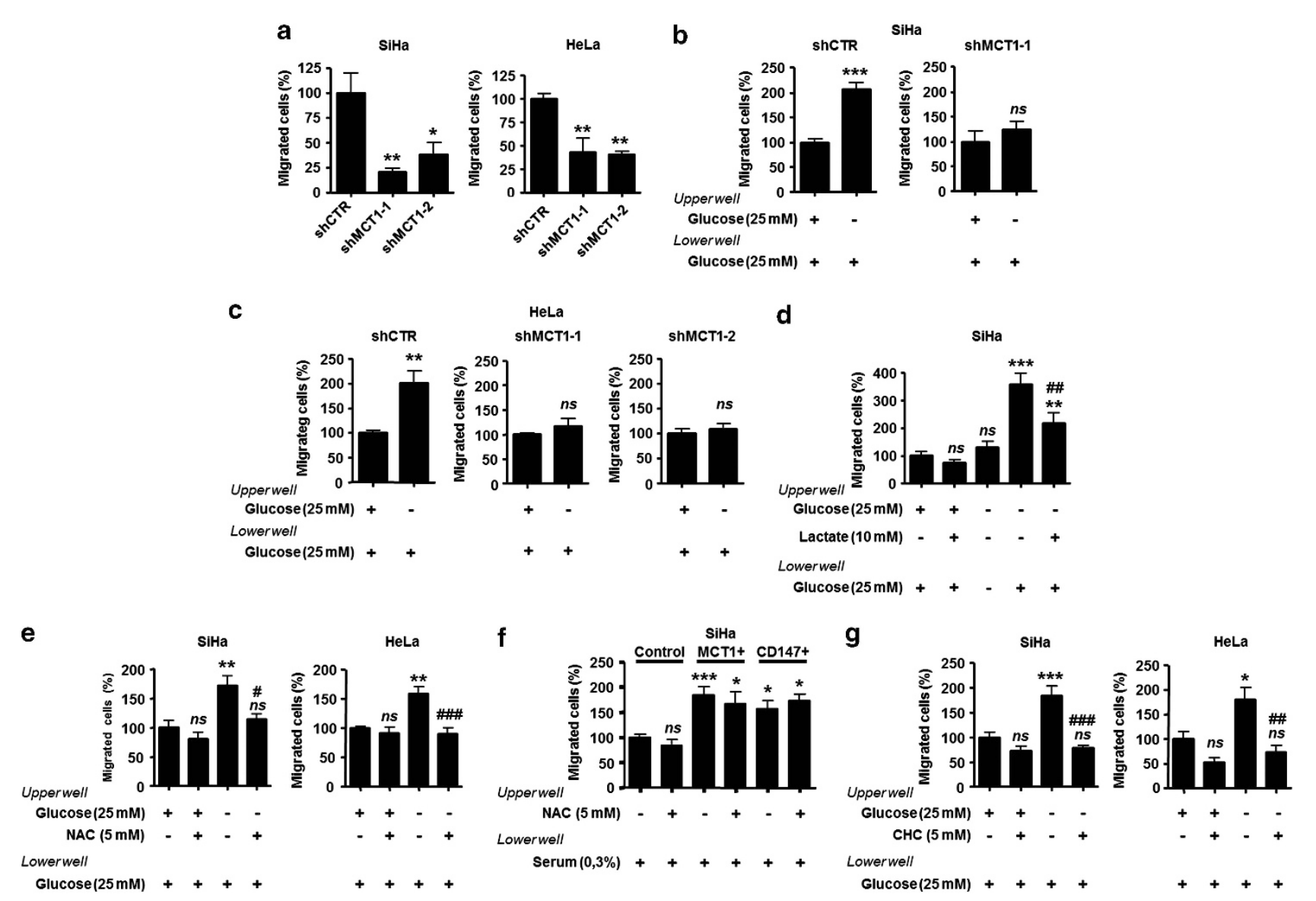

Figure 6. Targeting MCT1 inhibits tumor cell migration towards serum and glucose. $(\mathbf{a}-\mathbf{g})$ TC migration was studied in overnight transwell assays and quantified by counting migrated cells. (a) SiHa (left, $n=5-6$ ) and HeLa (right, $n=9-14$ ) cells infected with a control shRNA (shCTR) or 2 different specific shRNAs against MCT1 (shMCT1-1 and shMCT1-2) migrated towards serum. Migrated cell count is expressed as \% of shCTR $\left({ }^{*} P<0.05,{ }^{*} P<0.01\right.$ compared to shCTR). (b) The migration of SiHa cells infected with shCTR or shMCT1-1 was assayed with the indicated glucose supplementation to upper and lower wells. Migrated cell count is expressed as \% of basal migration (ns, not significant; ***P<0.005; $n=9-11$ ). (c) As in (b) but using HeLa cells transfected with shCTR, shMCT1-1 or shMCT1-2 ( $n$ s, not significant; ** $P<0.01 ; n=4-6$ ). (d) SiHa cell migration was assayed with the indicated supplementations to upper and lower wells. Migrated cell count is expressed as \% of basal migration (i.e., when glucose was present in both wells) (ns, not significant, ${ }^{* *} P<0.01,{ }^{* *} P<0.005$ compared to basal migration; ${ }^{\# \# P} P<0.01$ compared to migration from the glucose-starved to the glucose-containing wells; $n=6-13$ ). (e) SiHa (left, $\left.n=6\right)$ and HeLa (right, $n=4)$ cell migration was assayed with glucose or without glucose in the lower well and with $N$-acetyl-L-cysteine (NAC) in the upper well where indicated (ns, not significant, ${ }^{* *} P<0.01$ compared to basal migration; ${ }^{\#} P<0.01$, ${ }^{\# \# \#} P<0.005$ compared to migration from the glucose-starved to the glucose-containing wells). (f) SiHa cells were sham transfected (control) or transfected with a plasmid encoding SLC16A1/MCT1 $(\mathrm{MCT} 1+)$ or CD147 $(\mathrm{CD} 147+)$. Cell migration was assayed in the presence of glucose in both wells, with and without NAC in the upper well where indicated ( $n s$, not significant, ${ }^{*} P<0.05,{ }^{* *} P<0.005$ compared to migration of control cells without NAC in the upper wells; $n=4-11$ ). (g) as in (e) but with the MCT1 inhibitor $\alpha$-cyano-4-hydroxycinnamate $(\mathrm{CHC})$ in the upper well instead of NAC where indicated (ns, not significant ${ }^{*} P<0.05$, ${ }^{* * *} P<0.005$ compared to basal migration; ${ }^{\# \#} P<0.01$, ${ }^{\# \#} P<0.005$ compared to migration from the glucose-starved to the glucosecontaining wells; $n=6$ all).

scavenger mitoTEMPO ${ }^{23}$ and of the glutathione analogue NAC further revealed mitochondria-derived $\mathrm{H}_{2} \mathrm{O}_{2}$ as an important mediator of the phenotype. Inhibition was not complete though, suggesting the existence of additional pathways impacting MCT1CD147 stability (potentially including those active upon glutamine deprivation $)^{33}$ that would nevertheless exert a minor contribution under glucose deprivation as in this case NAC almost totally blocked TC migration (Figure 6e). In other circumstances, ROS could be bypassed. Indeed, overexpressing MCT1-CD147 experimentally was sufficient to promote TC migration in a ROSindependent manner (Figure 6f). As CD147 beyond MCT1 associates with a number of partners (including MCT4) to form supercomplexes, $^{39}$ additional and perhaps more indirect influences cannot be excluded. Furthermore, additional controls would be involved under hypoxia, ${ }^{40-42}$ explaining why hypoxia can fail to induce or can even repress MCT1 expression in vivo ${ }^{3,43}$ whereas it stimulates MCT1 expression in vitro ${ }^{44}$ (Figure 2a), and why we detected increased MCT1 expression at early time points (Figure 2a, $24 \mathrm{~h}$ ) whereas others did not find MCT1 induction upon a longer exposure to hypoxia $(48 \mathrm{~h}) .^{5}$ Modulating ROS can indeed impact the response of MCT1 to hypoxia (Supplementary Figure 4A).

Interestingly, heterocomplexes of CD147 with MCT4 (primarily involved in monocarboxylate export) ${ }^{4,5}$ have been previously reported to colocalize with $\beta 1$-integrin at the leading edge lamellipodia of migrating cells, ${ }^{45}$ supporting a role of MCTs in migration. Here, we documented that MCT1-CD147 complexes that accumulate in glucose-deprived cells are present among other locations in such protrusions (Figure 5 and Supplementary Figure 5) and regulate the migratory phenotype (Figure 6). Two main modes of action can be envisioned. On the one hand, within a gradient of glucose but no exogenous lactate, MCT1-expressing 
TCs will tend to increase their intracellular $\mathrm{pH}$ while decreasing the extracellular $\mathrm{pH}$ in the subcellular locations where MCT1 is expressed. This in turn may facilitate the activity of pro-migratory and pro-invasive enzymes and transporters, including matrix metalloproteinases. ${ }^{46,47}$ On the other hand, the MCT1-CD147 complex is known to behave as a scaffold for other proteins involved in cell migration and invasion. ${ }^{48}$ The composition of these supercomplexes is cell type-dependent and could logically be influenced by the cell environment and MCT1 expression and activity (conformation change).

In conclusion, our study shows that TCs in low glucose accumulate stabilized and functional MCT1-CD147 complexes that facilitate TC migration towards glucose. This paradigm offers a rationale for clinical data associating high MCT1 and CD147 expression to increased metastatic burden ${ }^{10,14,32}$ and for the metastatic phenotype promoted by therapies limiting glucose availability in tumors. ${ }^{31}$ We propose MCT1 inhibition as a pharmacological option to counter these effects.

\section{MATERIALS AND METHODS}

Cells and reagents

SiHa human cervix squamous cell carcinoma and HeLa human epithelial cervix cancer cells (ATCC, Manassas, VA, USA) were routinely cultured in DMEM containing D-glucose $(4,5 \mathrm{~g} / \mathrm{l})$, FBS $(10 \%)$ and penicillin-streptomycin (1\%). SiHa clones A and B were isolated from the wild-type cell population by using limiting dilution. Their glucose consumption and lactate production were quantified in clear cell lysates using specific enzymatic assays on a CMA600 analyzer (CMA Microdialysis AB, Solna, Sweden). Unless specified otherwise, all experiments were carried out in glutaminepyruvate-, lactate- and serum-free media buffered at $\mathrm{pH} 7.4(3.7 \mathrm{~g} / \mathrm{l}$ $\mathrm{NaHCO}_{3}, 5 \% \mathrm{CO}_{2}$ ). Glucose-deprived DMEM was from Sigma (Diegem, Belgium, catalogue \#D5030) and reconstituted without glucose, glutamine and serum where indicated. Hypoxia $\left(1 \% \mathrm{O}_{2}\right)$ was achieved in a Ruskinn hypoxic workstation (Bridgend, UK). Cell viability was systematically assessed using the NucleoCounter device from ChemoMetec (Lillerod, Denmark). MitoTempo was from Santa Cruz (Boechout, Belgium). Unless stated otherwise, all other reagents were from Sigma.

\section{Western blotting and immunostainings}

For immunoblotting, the lysates of an equal number of viable cells were separated on SDS-PAGE and transferred onto PVDF membranes with a semi-dry transfer system (Biorad, Nazareth, Belgium). Primary antibodies were a rabbit polyclonal against MCT1 (Millipore, Overijse, Belgium), a mouse monoclonal against CD147 (BD, Erembodegem, Belgium) and a mouse monoclonal against $\beta$-actin (Sigma). The protocol used for immunocytochemistry has been described previously. ${ }^{3}$ DAPI $\left(4^{\prime}\right.$, 6-diamidino-2-phenylindole) and Alexa fluor 488 phalloidin (Invitrogen, Gent, Belgium) were used to stain cell nuclei and the F-actin cytoskeleton, respectively. The in situ detection of MCT1-CD147 interaction was achieved using a proximity ligation assay (Duolink, Olink Bioscience, Uppsala, Sweden) according to the manufacturer's recommendations on cells fixed with $4 \%$ paraformaldehyde and permeabilized with Triton X-100 (0.1\%). Negative controls were run in the absence of the anti-CD147 primary antibody. All images were acquired with the same settings on a Zeiss Axiolmager microscope equipped with an Apotome module (Zeiss, Zaventem, Belgium). The relative frequency of occurrences was determined with the BlobFinder image analysis software (Centre for Image Analysis, Uppsala University, Sweden).

\section{Superoxide measurements}

MitoSOX Red, a mitochondria-selective fluorescent indicator, was used to specifically detect the superoxide anion ${ }^{22}$ according to the manufacturer's instructions. Signals were measured in 50000 cells on FACSCalibur (BD). The data were presented in the FL2 channel $(585 / 42 \mathrm{~nm})$ and analyzed with the FlowJo software (Tree Star, Ashland, OR, USA).

\section{RT-PCR}

Samples were collected from an equal amount of intact cells in Tri-Reagent (Molecular Research Center). RNA was recovered after separation in 1-bromo-3-chloropropane and precipitation with isopropanol, washed with ethanol (70\%) and resuspended in RNAse-free water. It was quantified using spectrophotometry (Nanodrop 1000, Thermo Scientific, Erembodegem, Belgium). Reverse transcription was performed on total RNA using the RevertAid M-MuLV Reverse Transcriptase and random hexamers (Fermentas, Erembodegem, Belgium). For quantitative real time amplification, SYBR green was used on a Biorad IQ5 system. Primers were: hMCT1 sense, 5'-GTGGCTCAGCTCCGTATTGT-3', antisense, 5'-GAGCCGACCTA AAAGTGGTG-3'; hCD147 sense, 5'-TGCTGGTCTGCAAGTCAGAG-3', antisense, 5'-GCGAGGAACTCACGAAGAAC-3'; housekeeping human ribosomal protein L19 (hRPL19) sense, 5'-CAAGCGGATTCTCATGGAACA-3', antisense, 5'-TGGTCAGCCAGGAGCTTCTT-3'.

\section{RNA interference and transfection}

Lentiviral infections were performed using a previously disclosed protocol. ${ }^{49}$ MCT1 shRNA (shMCT1) was purchased from Open Biosystems (Erembodegem, Belgium). Two vectors were used: TRCN0000038340 (shMCT1-1) and TRCN0000038339 (shMCT1-2). Control shRNA (shCTR) was Addgene plasmid 1864 (Cambridge, MA, USA). To obtain cells overexpressing MCT1 or CD147, we used the MCT1 CDNA expression vector pCMV6-Entry/mct1 (OriGene Technologies, Rockville, MD, USA) and full-length rat CD147 CDNA in a pCl-neo vector (kind gift of Professor J Pouysségur). After transfection with lipofectamine LTX (Invitrogen) according to the manufacturer's protocol, cells were selected on G418 and protein overexpression was verified by western blotting.

\section{Cell migration}

Cell migration assays were performed in a multiwell chemotaxis chamber (Neuro Probe, Leamington, UK) according to the manufacturer's instructions. Briefly, upper wells were loaded with 50000 cells in $50 \mu$ of medium containing no serum while the lower well always contained $0.1-0.3 \%$ of FBS in the same medium standardized for each batch according to basal migration activities. Where indicated, upper and/or lower wells were further supplemented with glucose, lactate or glutamine. After overnight incubation, migrated cells on the polycarbonate membrane interface were fixed with methanol and stained with crystal violet. They were counted under a microscope.

\section{Statistical analysis}

Data are expressed as mean \pm s.e.m. (error bars are sometimes smaller than symbols). Student's $t$ test, 1-way ANOVA (Bonferroni post-hoc test) or 2-way ANOVA (Bonferroni post-hoc test) were used where appropriate. $P<0.05$ was considered statistically significant.

\section{CONFLICT OF INTEREST}

The authors declare no conflict of interest.

\section{ACKNOWLEDGEMENTS}

This work was supported by the European Research Council (FP7/2007-2013 European Research Council Independent Researcher Starting Grant No. 243188 TUMETABO to PS), Interuniversity Attraction Pole grant \#UP7-03 from the Belgian Science Policy Office (Belspo), an Action de Recherche Concertée from the Communauté Française de Belgique (ARC 09/14-020 to PS and OF), the Fondation Belge contre le Cancer, the Fonds National de la Recherche Scientifique (FRS-FNRS), the Fonds National de la Recherche Scientifique Médicale, and the Télévie. OF is a Honorary Research Director and PS is a Research Associate of the FRS-FNRS. CDD is a Télévie Research Fellow. Funding bodies had no role in the design of the study, collection and analysis of data and decision to publish. The authors thank Prof Jacques Pouysségur (University of Nice Sophia Antipolis, France) for the kind gift of the CD147 vector, and Elise Beneteau, Morgane Tardy, Fabien Rodriguez, Thibaut Vazeille and Floriane Ribeiro for excellent technical assistance. This paper is dedicated to the loving memory of Mrs Anne-Sophie Brouckaert, PharmD.

\section{REFERENCES}

1 Porporato PE, Dadhich RK, Dhup S, Copetti T, Sonveaux P. Anticancer targets in the glycolytic metabolism of tumors: a comprehensive review. Front Pharmacol 2011; 2: 49.

2 Dhup S, Dadhich RK, Porporato PE, Sonveaux P. Multiple biological activities of lactic acid in cancer: influences on tumor growth, angiogenesis and metastasis. Curr Pharm Des 2012; 18: 1319-1330. 
3 Sonveaux P, Vegran F, Schroeder T, Wergin MC, Verrax J, Rabbani ZN et al. Targeting lactate-fueled respiration selectively kills hypoxic tumor cells in mice. J Clin Invest 2008; 118: 3930-3942.

4 Dimmer KS, Friedrich B, Lang F, Deitmer JW, Broer S. The low-affinity monocarboxylate transporter MCT4 is adapted to the export of lactate in highly glycolytic cells. Biochem J 2000; 350: 219-227.

5 Ullah MS, Davies AJ, Halestrap AP. The plasma membrane lactate transporter MCT4, but not MCT1, is up-regulated by hypoxia through a HIF-1alpha-dependent mechanism. J Biol Chem 2006; 281: 9030-9037.

6 Vegran F, Boidot R, Michiels C, Sonveaux P, Feron O. Lactate influx through the endothelial cell monocarboxylate transporter MCT1 supports an NF-kappaB/IL-8 pathway that drives tumor angiogenesis. Cancer Res 2011; 71: 2550-2560.

7 Sonveaux P, Copetti T, De Saedeleer CJ, Vegran F, Verrax J, Kennedy KM et al. Targeting the lactate transporter MCT1 in endothelial cells inhibits lactateinduced HIF-1 activation and tumor angiogenesis. PLoS ONE 2012; 7: e33418.

8 De Saedeleer CJ, Copetti T, Porporato PE, Verrax J, Feron O, Sonveaux P. Lactate Activates HIF-1 in Oxidative but Not in Warburg-Phenotype Human Tumor Cells. PLOS ONE 2012; 7: e46571.

9 Lu H, Forbes RA, Verma A. Hypoxia-inducible factor 1 activation by aerobic glycolysis implicates the Warburg effect in carcinogenesis. J Biol Chem 2002; 277: 23111-23115.

10 Walenta S, Mueller-Klieser WF. Lactate: mirror and motor of tumor malignancy. Semin Radiat Oncol 2004; 14: 267-274.

11 Halestrap AP, Wilson MC. The monocarboxylate transporter family--role and regulation. IUBMB Life 2012; 64: 109-119.

12 Asada K, Miyamoto K, Fukutomi T, Tsuda H, Yagi Y, Wakazono K et al. Reduced expression of GNA11 and silencing of MCT1 in human breast cancers. Oncology 2003; 64: 380-388

13 Kirk P, Wilson MC, Heddle C, Brown MH, Barclay AN, Halestrap AP. CD147 is tightly associated with lactate transporters MCT1 and MCT4 and facilitates their cell surface expression. EMBO J 2000; 19: 3896-3904.

14 Kennedy KM, Dewhirst MW. Tumor metabolism of lactate: the influence and therapeutic potential for MCT and CD147 regulation. Future Oncol 2010; 6 : 127-148.

15 Le Floch R, Chiche J, Marchiq I, Naiken T, Ilk K, Murray CM et al. CD147 subunit of lactate/H + symporters MCT1 and hypoxia-inducible MCT4 is critical for energetics and growth of glycolytic tumors. Proc Natl Acad Sci USA 2011; 108 16663-16668.

16 Walenta S, Snyder S, Haroon ZA, Braun RD, Amin K, Brizel D et al. Tissue gradients of energy metabolites mirror oxygen tension gradients in a rat mammary carcinoma model. Int J Radiat Oncol Biol Phys 2001; 51: 840-848.

17 Hirayama A, Kami K, Sugimoto M, Sugawara M, Toki N, Onozuka H et al. Quantitative metabolome profiling of colon and stomach cancer microenvironment by capillary electrophoresis time-of-flight mass spectrometry. Cancer Res 2009; 69: 4918-4925.

18 Reitzer LJ, Wice BM, Kennell D. Evidence that glutamine, not sugar, is the major energy source for cultured HeLa cells. J Biol Chem 1979; 254: 2669-2676.

19 Wu R, Racker E. Regulatory mechanisms in carbohydrate metabolism. IV. Pasteur effect and Crabtree effect in ascites tumor cells. J Biol Chem 1959; 234 1036-1041.

20 Yun J, Rago C, Cheong I, Pagliarini R, Angenendt P, Rajagopalan $\mathrm{H}$ et al. Glucose deprivation contributes to the development of KRAS pathway mutations in tumor cells. Science 2009; 325: 1555-1559.

21 Murphy MP. How mitochondria produce reactive oxygen species. Biochem J 2009 417: 1-13.

22 Mukhopadhyay P, Rajesh M, Hasko G, Hawkins BJ, Madesh M, Pacher P. Simultaneous detection of apoptosis and mitochondrial superoxide production in live cells by flow cytometry and confocal microscopy. Nat Protoc 2007; 2: 2295-2301.

23 Dikalova AE, Bikineyeva AT, Budzyn K, Nazarewicz RR, McCann L, Lewis W et al. Therapeutic targeting of mitochondrial superoxide in hypertension. Circ Res 2010; 107: $106-116$.

24 Schewe T. Molecular actions of ebselen--an antiinflammatory antioxidant. Gen Pharmacol 1995; 26: 1153-1169.

25 Mattila PK, Lappalainen P. Filopodia: molecular architecture and cellular functions. Nat Rev Mol Cell Biol 2008; 9: 446-454.

26 Manning Fox JE, Meredith D, Halestrap AP. Characterisation of human monocarboxylate transporter 4 substantiates its role in lactic acid efflux from skeletal muscle. J Physiol 2000; 529: 285-293.
27 Wallace DC. Mitochondria and cancer. Nat Rev Cancer 2012; 12: 685-698.

28 Dang CV, Hamaker M, Sun P, Le A, Gao P. Therapeutic targeting of cancer cell metabolism. J Mol Med (Berl) 2011; 89: 205-212.

29 Eyster CA, Higginson JD, Huebner R, Porat-Shliom N, Weigert R, Wu WW et al. Discovery of new cargo proteins that enter cells through clathrin-independent endocytosis. Traffic 2009; 10: 590-599.

30 Smith JP, Uhernik AL, Li L, Liu Z, Drewes LR. Regulation of Mct1 by CAMPdependent internalization in rat brain endothelial cells. Brain Res 2012; 1480 $1-11$

31 De Bock K, Mazzone M, Carmeliet P. Antiangiogenic therapy, hypoxia, and metastasis: risky liaisons, or not? Nat Rev Clin Oncol 2011; 8: 393-404.

32 Lee GH, Kim DS, Chung MJ, Chae SW, Kim HR, Chae HJ. Lysyl oxidase-like-1 enhances lung metastasis when lactate accumulation and monocarboxylate transporter expression are involved. Oncol Lett 2011; 2: 831-838.

33 Cardaci S, Rizza S, Filomeni G, Bernardini R, Bertocchi F, Mattei M et al Glutamine deprivation enhances antitumor activity of 3-bromopyruvate through the stabilization of monocarboxylate transporter-1. Cancer Res 2012; 72 : 4526-4536

34 Birsoy $\mathrm{K}$, Wang $\mathrm{T}$, Possemato R, Yilmaz $\mathrm{OH}$, Koch $\mathrm{CE}$, Chen WW et al. MCT1mediated transport of a toxic molecule is an effective strategy for targeting glycolytic tumors. Nat Genet 2012; 45: 104-108.

35 Ferretti AC, Larocca MC, Favre C. Nutritional stress in eukaryotic cells: oxidative species and regulation of survival in time of scarceness. Mol Genet Metab 2012; 105: 186-192.

36 Jia L, Xu H, Zhao Y, Jiang L, Yu J, Zhang J. Expression of CD147 mediates tumor cells invasion and multidrug resistance in hepatocellular carcinoma. Cancer Invest 2008; 26: 977-983.

37 Izumi H, Takahashi M, Uramoto H, Nakayama Y, Oyama T, Wang KY et al. Monocarboxylate transporters 1 and 4 are involved in the invasion activity of human lung cancer cells. Cancer Sci 2011; 102: 1007-1013.

38 Li J, Peng L, Wu L, Kuang Y, Su J, Yi M et al. Depletion of CD147 sensitizes human malignant melanoma cells to hydrogen peroxide-induced oxidative stress. J Dermatol Sci 2010; 58: 204-210.

39 lacono KT, Brown AL, Greene Ml, Saouaf SJ. CD147 immunoglobulin superfamily receptor function and role in pathology. Exp Mol Pathol 2007; 83: 283-295.

40 Boidot R, Vegran F, Meulle A, Le Breton A, Dessy C, Sonveaux P et al. Regulation of monocarboxylate transporter MCT1 expression by p53 mediates inward and outward lactate fluxes in tumors. Cancer Res 2012; 72: 939-948.

41 Ke X, Fei F, Chen Y, Xu L, Zhang Z, Huang Q et al. Hypoxia upregulates CD147 through a combined effect of HIF-1alpha and Sp1 to promote glycolysis and tumor progression in epithelial solid tumors. Carcinogenesis 2012; 33: 1598-1607.

42 Merezhinskaya N, Fishbein WN. Monocarboxylate transporters: past, present, and future. Histol Histopathol 2009; 24: 243-264.

43 Rademakers SE, Lok J, van der Kogel AJ, Bussink J, Kaanders JH. Metabolic markers in relation to hypoxia; staining patterns and colocalization of pimonidazole, HIF-1alpha, CAIX, LDH-5, GLUT-1, MCT1 and MCT4. BMC Cancer 2011; 11: 167.

44 Cheng $\mathrm{C}$, Edin NF, Lauritzen $\mathrm{KH}$, Aspmodal I, Christoffersen $\mathrm{S}$, Jian $\mathrm{L}$ et al. Alterations of monocarboxylate transporter densities during hypoxia in brain and breast tumour cells. Cell Oncol 2012; 35: 217-227.

45 Gallagher SM, Castorino JJ, Philp NJ. Interaction of monocarboxylate transporter 4 with beta1-integrin and its role in cell migration. Am J Physiol Cell Physiol 2009; 296: C414-C421.

46 Stock C, Schwab A. Protons make tumor cells move like clockwork. Pflugers Arch 2009; 458: 981-992.

47 Webb BA, Chimenti M, Jacobson MP, Barber DL. Dysregulated pH: a perfect storm for cancer progression. Nat Rev Cancer 2011; 11: 671-677.

$48 \mathrm{Xu} \mathrm{D}$, Hemler ME. Metabolic activation-related CD147-CD98 complex. Mol Cell Proteomics 2005; 4: 1061-1071.

49 Moon EJ, Sonveaux P, Porporato PE, Danhier P, Gallez B, Batinic-Haberle I et al. NADPH oxidase-mediated reactive oxygen species production activates hypoxiainducible factor-1 (HIF-1) via the ERK pathway after hyperthermia treatment. Proc Natl Acad Sci USA 2010; 107: 20477-20482.

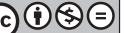

This work is licensed under a Creative Commons AttributionNonCommercial-NoDerivs 3.0 Unported License. To view a copy of this license, visit http://creativecommons.org/licenses/by-nc-nd/3.0/

Supplementary Information accompanies this paper on the Oncogene website (http://www.nature.com/onc) 